\title{
Par-delà la reconnaissance. L'attention comme paradigme pour une éthique asymétrique
}

\section{Emmanuel Alloa}

\section{(2) OpenEdition}

1 Journals

Édition électronique

URL : http://journals.openedition.org/alter/1618

DOI : 10.4000/alter.1618

ISSN : 2558-7927

Éditeur :

Association ALTER, Archives Husserl (CNRS-UMR 8547)

\section{Édition imprimée}

Date de publication : 1 octobre 2010

Pagination : 125-141

ISBN : 2-9522374-6-8

ISSN : $1249-8947$

\section{Référence électronique}

Emmanuel Alloa, «Par-delà la reconnaissance. L'attention comme paradigme pour une éthique asymétrique », Alter [En ligne], 18 | 2010, mis en ligne le 01 juin 2020, consulté le 29 juin 2020. URL http://journals.openedition.org/alter/1618 ; DOI : https://doi.org/10.4000/alter.1618 


\title{
PAR-DELÀ LA RECONNAISSANCE. L'ATTENTION COMME PARADIGME POUR UNE ÉTHIQUE ASYMÉTRIQUE
}

\author{
Emmanuel Alloa
}

\section{1. Éthiques de la reconnaissance. La scène hégélienne}

L'attention n'est pas le seul concept épistémologique à être aujourd'hui invoqué dans un contexte éthique. Un autre concept, ancré lui aussi dans une tradition de la théorie de la connaissance, domine aujourd'hui les débats contemporains sur l'éthique et la politique : le concept de reconnaissance. Les crises sociales récentes (comme par exemple les insurrections dans les banlieues françaises en 2005) ont souvent été interprétées comme autant de crises de la reconnaissance: une partie de la société continue à faire les frais d'une non-reconnaissance sociétale, ou encore, elle ne se reconnaît pas dans l'identité universaliste et donc anonyme que lui confère un régime républicain, ce qui revient substantiellement au même. "La lutte pour la reconnaissance, » résume Nancy Fraser, « est en train de devenir rapidement la forme paradigmatique du conflit politique ${ }^{1} »$.

La nécessité de repenser la reconnaissance des certains groupes sociaux est discutée depuis longtemps dans l'aire anglo-américaine. Pour Charles Taylor, l'avènement des sociétés multiculturelles conduit inévitablement à une nouvelle "politique de reconnaissance ${ }^{2}$ ». Selon Taylor, il s'agit de rendre compte du fait que les identités politiques ne sauraient se résumer à

1. Nancy Fraser, Qu'est-ce que la justice sociale ? Reconnaissance et redistribution, trad. Estelle Ferrarese, Paris, La Découverte, 2005 (traduction modifiée).

2. Charles Taylor, "Politics of recognition », in Multiculturalism and "The Politics of Recognition ", sous la dir. de Amy Gutman, Princeton, Princeton University Press, 1992, p. 25-73 («La politique de reconnaissance ", Multiculturalisme, différence et démocratie, avec des commentaires de Amy Gutmann, Steven C. Rockefeller, Michael Walzer et Susan Wolf, trad. Denis-Armand Canal, Paris, Flammarion 1994, p. 41-99). 


\section{L'attention}

la citoyenneté abstraite que propose l'universalisme, mais que les sujets se définissent également par l'appartenance à certaines groupes définis par des critères ethniques, linguistiques, sexuels ou religieux, revendiquant une reconnaissance au niveau politique. La réhabilitation du concept hégélien d'une «lutte pour la reconnaissance » (le Kampf um Anerkennung), proposé par des auteurs aussi divers que Taylor, Nancy Fraser, Axel Honneth, Judith Butler, Emmanuel Renault, Jean-Marc Ferry ou Paul Ricœur ${ }^{3}$, abandonne les discussions sur les «voiles de l'ignorance » et autres perfectionnements des théories procédurales vers le champ concret des revendications sociales. Tandis que les théories politiques des décennies précédentes restaient placées sous le signe d'une éthique de la redistribution, c'est à présent l'exigence de reconnaissance identitaire et les luttes qui en découlent qui se retrouvent au cœur des débats ${ }^{4}$.

La fortune du concept de reconnaissance dérive indéniablement du fait qu'il rapproche la théorie politique des conflits réels. L'idée de justice n'est plus pensée comme c'était le cas précédemment à partir d'une idée régulative qui gouvernerait la redistribution des biens (Rawls), mais en fonction de revendications s'enracinant dans l'expérience d'une injustice subie et d'un tort vécu. Il y a toutefois des bonnes raisons pour croire que le concept de reconnaissance ne permet pas de rendre compte de l'intégralité des rapports intersubjectifs ou encore, et plus fondamentalement, que la réflexion sur l'intersubjectivité ne pourra inévitablement qu'être faussée si elle est ramenée à la scène primitive de la reconnaissance.

Nous évoquerons dans la première partie de cet essai trois objections que l'on peut adresser au paradigme de la reconnaissance: la présupposition épistémologique (1.1), la présupposition symétrique (1.2) et la présupposition dyadique (1.2). Dans la seconde partie, nous proposerons l'ébauche d'une alternative à ce paradigme : une éthique de l'inter-attention (2) qui ne serait $n i$ épistémocentrique ni symétrique ni dyadique. En mettant en regard certaines réflexions phénoménologiques sur l'attention avec les études empiriques sur la joint attention («attention conjointe»), on esquissera l'horizon d'une éthique phénoménologique qui se délivre du primat de l'objet (2.1), de la personne (2.2) et de la propriété (2.3).

3. Axel Honneth, Kampf um Anerkennung. Zur moralischen Grammatik sozialer Konflikte, Francfort, Suhrkamp, 1992 (La lutte pour la reconnaissance. Grammaire morale des conflits sociaux, trad. Pierre Rusch, Paris, Cerf, 2000). Jean-Marc Ferry, L'éthique reconstructive, Paris, Cerf, 1996. Emmanuel Renault, Mépris social. Éthique et politique de la reconnaissance, Éditions du passant 2000. Paul Ricœur, Parcours de la reconnaissance, Paris, Stock, 2004. Emmanuel Renault, L'expérience de l'injustice. Reconnaissance et clinique de l'injustice, Paris, La Découverte, 2004. Alain Caillé (sous la dir.), La quête de reconnaissance. Nouveau phénomène social total, Paris, La Découverte, 2007. Pour une vue d'ensemble cf. Stéphane Haber «Hegel vu depuis la reconnaissance », in : Revue du MAUSS, «De la reconnaissance ", $\mathrm{n}^{\circ} 23,2004$, p. 70-87.

4. Pour suivre l'histoire de ce déplacement, cf. Nancy Fraser, Qu'est-ce que la justice sociale ? Reconnaissance et redistribution, Paris, La Découverte, 2005. Voir également le débat Nancy Fraser \& Axel Honneth, Recognition or Redistribution? New York, Verso, 2003. 
Le concept de « reconnaissance » (Anerkennung) est introduit par Hegel dans son Système de la vie éthique (System der Sittlichkeit ${ }^{5}$ ). Dans cet écrit de 1802/1803, on trouve l'ébauche des fondements de la philosophie pratique, et notamment l'idée d'une dialectique entre maître et esclave. Si c'est cette dernière qui est seule retenue dans la Phénoménologie de l'esprit, on ne peut cependant la comprendre sans le concept de reconnaissance qui en constitue l'arrière-fond. À travers une discussion avec Fichte (le System der Sittlichkeit est sous-titré «Critique du droit naturel fichtéen »), c'est pourtant encore Kant qui est visé.

1) Pour Hegel, le fondement du concept de socialité chez Kant - le respect - reste un modèle encore trop intellectualiste. Le concept de reconnaissance marquera une tentative pour faire dériver historiquement et généalogiquement les forces sociales en jeu dans le réel.

2) L'égalité devant la loi postulée par Kant et dont la notion de respect dérive ne rend pas compte, pour Hegel, de l'inégalité existant de fait. Quand on analyse des relations de proximité au sein de la vie éthique (Sittlichkeit) comme par exemple les rapports parents-enfants ou maître-esclave, on ne peut que constater, avant même l'identité de droit garantie par la socialité de l'Etat, des situations d'asymétrie. Une théorie de la reconnaissance prétend donc rendre compte des inégalités de fait régnant dans les rapports intersubjectifs.

3) Bien qu'enraciné dans la situation modèle qui est celle d'un rapport de proximité, la lutte pour la reconnaissance excède selon Hegel la sphère dyadique de Fichte. La lutte pour la reconnaissance détermine également les rapports au sein de l'état de droit et elle débouche sur une lutte pour la reconnaissance de groupes sociaux.

Dans ce qui suit, il s'agira de montrer dans quelle mesure les trois présuppositions (épistémologiques, symétriques et dyadiques) demeurent prégnantes dans le modèle hégélien et, au-delà de Hegel, chez les auteurs qui font de la lutte pour la reconnaissance leur scène primitive.

\subsection{La présupposition épistémologique}

L'éthique kantienne, on l'a dit, fonde le principe de l'intersubjectivité sur le respect de la loi. La loi n'est pas le fruit d'une imposition par la force, son intuition dérive du sentiment moral (moralisches Gefühl) que ce qui impose une limite à notre amour-propre mérite notre respect. L'intuition d'un principe rationnel dépassant la sphère du Moi produit alors le respect pour autrui, non pas en vertu de la perfection de la personne, mais en vertu de son humanité. La rencontre intersubjective produit ainsi une « limitation de notre amour-propre par la dignité de l'humanité dans une autre personne »

5. G.W.F. Hegel, System der Sittlichkeit [1802/03], Werke 2 : Jenaer Schriften 1802-1807, éd. Eva Moldenhauer et Karl Markus Michel, Francfort, Suhrkamp, 1970 (Système de la vie éthique, trad. J. Taminiaux, Paris, Payot, 1976) 


\section{L'attention}

(Einschränkung unserer Selbstschätzung durch die Würde der Menschheit in einer anderen Person) ${ }^{6}$. Par l'intuition de la rationalité de la loi, c'est l'épanouissement de toute personne qui se voit garanti, dans la mesure où elle impose la maxime selon laquelle tout agir doit traiter « l'humanité aussi bien dans ta personne que dans la personne de tout autre toujours en même temps comme une fin, et jamais simplement comme un moyen ${ }^{7}$ ». Le respect pour autrui est donc basé originairement sur la «reconnaissance d'une dignité (dignitas) dans les autres hommes, c'est-à-dire d'une valeur, qui n'a pas de prix, pas d'équivalent, contre lequel le prix de l'æstimation (stimii) pourrait être échangé8. »

Selon Hegel - ou du moins le Hegel des premiers écrits d'Iéna - une telle position concède encore trop à la rationalité d'un sujet autonome, alors qu'en réalité, l'espace de la moralité des mœurs se rapproche plus de la lutte hobbesienne de tous contre tous qu'à la contrainte non contraignante (zwanglose Zwang) de la loi kantienne ${ }^{9}$. La différence vis-à-vis de Hobbes concerne ici une interprétation différente de l'état de nature pour laquelle Hegel s'inspire de Fichte : le conflit qui oppose les individus ne relève pas de la satisfaction d'une pulsion de survie, mais indique la dépendance réciproque des sujets. Le sujet assujetti par un autre découvre que ce dernier n'use guère de la violence en raison d'un quelconque besoin physique, mais qu'il est mû par un désir de reconnaissance que seul l'autre sujet peut lui conférer, et fût-ce dans l'assujettissement. En ce sens, toute conscience de soi dépend préalablement d'une reconnaissance par l'autre dans lequel le Moi se reflète et inversement.

Axel Honneth a montré comment la découverte de la dépendance du sujet (qu'on peut dater de l'époque de la Realphilosophie de Iéna) se verra progressivement reprise et relevée dans le cadre d'une philosophie de la conscience. Celle-ci conduit inévitablement à la spiritualisation de la reconnaissance et, par extension, à son épistémologisation. Dès le Système de la Philosophie spéculative de 1803/1804, la reconnaissance est instanciée comme une étape sur le chemin de l'auto-connaissance de la conscience, si bien que la reconnaissance se verra à présent définie comme démarche par laquelle une conscience, tendant abstraitement à la totalité, se connaît «dans

6. Emmanuel Kant, Metaphysische Anfangsgründe der Tugendlehre. Metaphysik der Sitten. Zweiter Teil, Akademie-Ausgabe (=AA), Berlin, De Gruyter, 1968, t. VI, p. 449 (Doctrine de la vertu. Métaphysique des mours. Deuxième partie, trad. Alexis Philonenko, Paris, Vrin 1985, p. 127, traduction modifiée).

7. Emmanuel Kant, Grundlegung der Metaphysik der Sitten, Werke, AA t. IV, p. 429 (fr. Fondement de la métaphysique des mours, CEuvres philosophiques, Paris, Gallimard, «Pléiade», 1985, t. II, p. 295).

8. Kant, Metaphysik der Sitten, op. cit., AA t. VI, p. 462 (Métaphysique des mœurs, op. cit., p. 140)

9. Sur l'importance de Hobbes dans l'élaboration de la « lutte pour la reconnaissance», cf. Ludwig Siep : « Der Kampf um Anerkennung bei Hegel. Zu Hegels Auseinandersetzung mit Hobbes in den Jenaer Schriften », in : Hegel-Studien, t. 9 (1974), p. 155-207. 
une autre totalité semblable, une conscience, comme <étant $>$ elle-même ${ }^{10}$ ». Ce n'est qu'en tant qu'elle participe déjà de l'Esprit que la conscience est capable de reconnaissance ; c'est parce que dans sa partialité elle est capable de reconnaissance qu'elle peut accéder à la pleine connaissance, bref : la reconnaissance est ramenée, dès le Système de la Philosophie spéculative et plus clairement encore dès la Phénoménologie de l'Esprit, à une recognition.

Si Honneth a lui-même cherché à explorer des alternatives à la clôture épistémologique de la scène hégélienne, notamment en prolongeant ces réflexions en direction de George Herbert Mead et de la psychosociologie, d'autres théoriciens de la reconnaissance tombent sous le coup d'une réintellectualisation $\mathrm{du}$ concept, quand ils essayent de répondre à la question qu'ouvre tout acte de reconnaissance : quelle base commune y a-t-il pour permettre la reconnaissance mutuelle? Charles Taylor par exemple relit Hegel avec Kant et réintroduit l'idée d'un «bien constitutif» qui qualifie tout espace moral et fonde la reconnaissance mutuelle. Grâce au partage de tels biens constitutifs par une communauté, chaque sujet peut retrouver son authenticité au sein de cette communauté. S'il ne possède pas d'authenticité en dehors d'un échange dialogique, le sujet peut néanmoins accéder à son être propre au sein d'une communauté morale et culturelle, elle-même en dialogue avec d'autres communautés auxquelles il faudra accorder un «droit à la différence ». Tout en fustigeant l'intellectualisme de la tradition philosophique, en particulier des Lumières, auquel il reproche de ne pas avoir pensé que tout sujet est invariablement « engagé » dans le monde, l'engagement reste soumis chez Taylor à un modèle herméneutique et l'action à la compréhension. Reprenant l'idée gadamérienne de la fusion des horizons d'interprétation, la «compréhension engagée » envisagée par Taylor ${ }^{11}$ reste toute relative, car elle suppose le point de vue d'un juge qui se sait déjà du bon côté. Le sujet taylorien qui reconnaît la valeur de l'autre culture est toujours un sujet occidental et donc anonyme, un sujet qui ne saurait être remis en cause pour sa partialité, car il jouit, en tant que membre du projet de la modernité occidentale, malgré tout d'un " exceptionnalisme moral $^{12} »$. Dans son livre Beyond recognition, la philosophe américaine Kelly Oliver fait ainsi de Charles Taylor l'exemple même d'une théorie intellectualiste de la reconnaissance, d'autant plus problématique qu'elle reste inavouée, car se parant du couvert de multiculturalisme. Moins essentialiste que d'autres positions du camp communautaire aux Etats-Unis

10. G.W.F. Hegel, Jenaer Systementwürfe I. Das System der spekulativen Philosophie, Hambourg, Meiner, 1986, fragment 22, p. 189 (Le Premier Système. La philosophie de l'esprit 1803-1804, éd. et traduction Myriam Bienenstock, Paris, PUF, 1999, p. 88 - cette édition rend obsolète la traduction de Guy Planty-Bonjour La première philosophie de l'Esprit, de 1969, fondée encore sur le classement des fragments établi par Franz Rosenzweig).

11. Cf. l'auto-interprétation de Taylor dans Charles Taylor et l'interprétation de l'identité moderne, Centre culturel international de Cerisy-la-Salle, Presses Université Laval, 1998, p. 354.

12. Charles Taylor, Sources of the Self: The Making of the Modern Identity, Cambridge University Press, 1992, p. 397. 


\section{L'attention}

et insistant avec Hegel sur la part d'hétéronomie dans l'émergence des identités culturelles, son concept d'authenticité oscille néanmoins étrangement entre un usage constatif et un usage performatif. Tout en dénonçant toute authenticité donnée d'avance, Taylor maintient l'idée d'une existence authentique et non-aliénée qui procéderait d'un acte de recognition de sa propre particularité ${ }^{3}$.

\subsection{La présupposition symétrique}

S'appuyant sur cette conviction, d'autres théoriciens de la reconnaissance tels que Judith Butler ont pour leur part opté pour une lecture ouvertement performative du terme. Pour Butler, l'identité ne préexiste pas à la scène agonale sur laquelle les identités sont conférées, modifiées et défaites par des actes réciproques.

La leçon de la reconnaissance hégélienne - Judith Butler résume ainsi les lectures anti-essentialistes qu'elle fait de la Phénoménologie de l'Esprit - est qu'à travers les actes de reconnaissance qu'appelle mon existence sociale, «je deviens autre que ce que j'étais et ne peux donc plus revenir vers ce que j'étais ${ }^{14}$ ». Par sa lecture, Butler s'inscrit dans la filiation de l'interprétation $\mathrm{d}^{\prime}$ Alexandre Kojève ${ }^{15}$, tout en relevant judicieusement la désontologisation qu'opérait l'Introduction à la philosophie de Hegel: Kojève réduit la reconnaissance à la relation maître-esclave et dépouille ainsi la Phénoménologie de l'Esprit de son assise ontologique, pour ne plus la lire que comme une anthropologie. Si Butler insiste elle aussi sur le caractère anti-fondationnaliste et processuel, elle souligne également la réciprocité de toute reconnaissance chez Hegel, point précisément rejeté par Kojève ${ }^{16}$ : La conscience de soi ne peut avoir d'action sur l'autre conscience, sans en être inversement modifiée; reconnaître autrui revient à se faire reconnaître en retour. La reconnaissance de l'autre ressemble alors à un don qui, dans un effet de boomerang, me revient tellement vite que je peux me demander, avec Levinas, s'il a vraiment jamais quitté mes mains ${ }^{17}$. Qu'elle soit donc vraiment ecstatique comme le veut Butler ou non, la reconnaissance est en tout cas toujours réciproque et implique une confirmation mutuelle. Hegel ne fera que radicaliser cette perspective, quand vers la fin de la Phénoménologie de l'Esprit il pose en médiateur au sein duquel toute conscience se reconnaît comme identique à l'autre, un "Je, qui est un Nous, et un Nous qui est un Je » (Ich,

13. Kelly Oliver, Witnessing. Beyond recognition, Minneapolis, University of Minnesota Press, 2001, p. 44-46.

14. Judith Butler, Giving an Account of Oneself, New York, Fordham Press, 2005, p. 27.

15. Alexandre Kojève, Introduction à la Lecture de Hegel, Paris, Gallimard, 1947 (1983).

16. Judith Butler, Subjects of Desire: Hegelian Reflections in Twentieth-Century France, New York, 1987, p. 63.

17. Judith Butler, Giving an Account of Oneself, op. cit., p. 27. 
das Wir, und Wir, das Ich ist) ${ }^{18}$, médium où tout un chacun est relevé, mais également relevé de son être-ainsi.

Refusant cette synthèse ultime, la plupart des théoriciens de la reconnaissance assument néanmoins l'aspect réciproque et symétrique de la description hégélienne, faisant de la «reconnaissance entre égaux » la et tentant de ramener la réciprocité aux premiers stade«seule solution satisfaisante» au problème de la lutte pour la reconnaissance ${ }^{19} \mathrm{~s}$ de $l^{\prime}$ existence humaine ${ }^{20}$. La réciprocité serait ainsi le gage de l'épanouissement de tout un chacun, sans qu'il y ait de gagnants ni de perdants et qui permettrait, selon Axel Honneth par exemple, d'atteindre un « rapport à soi non brisé » (ungebrochenes Selbstverhältnis) ${ }^{21}$.

C'est précisément cette symétrie qui fait problème, comme le souligne le phénoménologue Bernhard Waldenfels dans sa critique du paradigme de la reconnaissance ${ }^{22}$. Une phénoménologie des actes de reconnaissance montre immédiatement que la symétrie n'est qu'une chimère contractualiste, alors que l'asymétrie entre déjà en jeu lorsqu'il s'agit de décider qui signera en premier un contrat. Plus fondamentalement, les processus de reconnaissance ne relèvent que rarement de l'ordre de la décision, car ne peut conférer de reconnaissance que celui ou celle qui aura préalablement été interpellé par une demande de reconnaissance. Reconnaître quelqu'un ou quelque chose « en tant que $\mathrm{x}$ » ou « $\mathrm{y}$ » suppose qu'il ait déjà fait irruption dans mon champ, qu'il ait suscité mon attention. La synchronie n'est, au mieux, qu'une rétrojection post festum, car la scène explicite de revendications réciproques est appelée par un événement qui ne survit, dans ce qu'il institue, que comme antécédence inaccessible. La cause initiale pour l'échange d'un salut amical est irrécupérable, elle dépend d'une microphysique de circonstances et ses modalités, son intensité et sa durée d'une myriade de facteurs pour lesquels toute formalisation protocolaire arriverait déjà invariablement trop tard. S'il y a une lutte pour la reconnaissance, c'est avant tout un différend sur ce qui demeure exclu du cadre légal du protocole; ce qui se fait entendre avec insistance, c'est ce qui n'a pas encore de voix dans l'échange codifié des arguments. En ce sens, si l'idée d'un appel à la reconnaissance a un sens, c'est que celui-ci précède le face-à-face symétrique des sujets constitués. On peut certes affirmer avec Martin Buber, et à sa suite avec Levinas, qu'autrui est premier par rapport au Moi, mais ce serait encore trop peu si l'on méprenait autrui pour un autre ego, tout juste plus précoce. C'est la situation dyadique elle-même, troisième présupposé du paradigme de la reconnaissance, qui révèle ainsi ses limites.

18. G.W. F. Hegel, Phänomenologie des Geistes, Werke 3, éd. Eva Moldenhauer et Karl Markus Michel, Francfort, Suhrkamp, 1970, p. 145 (Phénoménologie de l'esprit, trad. Jean-Pierre Lefebvre, Paris, Aubier, 1991, p. 149).

19. Charles Taylor, « Politics of recognition», art. cit., p. 50.

20. Honneth, Kampf um Anerkennung, op. cit., chap. 5 (La lutte pour la reconnaissance, op. cit., chap. 5).

21. Honneth, Kampf um Anerkennung, p. 196.

22. Bernhard Waldenfels, Schattenrisse der Moral, Francfort, Suhrkamp, 2006, p. 271sq. 


\section{L'attention}

\subsection{La présupposition dyadique}

Certains théoriciens de la reconnaissance tels que Jessica Benjamin pointent les apories d'une conception symétrique de la reconnaissance, mais situent encore toujours l'asymétrie au sein d'une relation duelle ou dyadique $^{23}$. Si l'on échappe au fantasme égalitaire, le principe dialogique, également postulé par d'autres auteurs tels que Charles Taylor ${ }^{24}$, reste déterminant et marginalise les instances du tiers. Dans une discussion avec Jessica Benjamin, Judith Butler démontre les limitations d'une telle perspective, car Benjamin pose comme origine une dyade qui n'est tout au mieux qu'un résultat intermédiaire et momentané. La relation dyadique n'émerge que sur fond de relations multiples et elle retourne ensuite $\mathrm{s}^{\prime} \mathrm{y}$ fondre. En partant comme le fait Benjamin d'une scène primitive du face-àface, « je reste donc au centre du Désir de l'autre et le narcissisme est, par définition, satisfait ${ }^{25} »$. Il s'agirait au contraire de comprendre que par définition, le désir excède toujours ce sur quoi il se porte (qu'il s'agisse d'une personne ou d'un objet) et qu'il dépasse toujours la dyade vers son dehors. Accepter que le désir consiste, avant tout, en un désir du désir de l'Autre signifie défaire l'étau de l'identification pour ouvrir les relations interpersonnelles à un réseau complexe de renvois jamais entièrement objectivables. "La dyade est un résultat et non une présupposition ${ }^{26}$ ».

En passant du légalisme des éthiques procédurales à l'intimisme de certaines nouvelles théories de la reconnaissance, ce sont toutes les instances tierces qui ont été sacrifiées. Or, par essence, la structure de la reconnaissance ne peut pas être dyadique, dès lors qu'elle implique inévitablement trois termes. À la différence du verbe « connaître »- et également à la différence du verbe « prêter attention »- le verbe « reconnaître » fait partie des verbes trivalents comme "remercier», "donner» ou encore "communiquer». Contrairement aux verbes bivalents pour lesquels un prédicat se voit associé à un sujet, les verbes trivalents impliquent trois termes ou actants. L'acte de reconnaissance ne saurait s'arrêter à la reconnaissance $\mathrm{d}^{\prime} y$ par $x ; x$ doit toujours reconnaître $y$ «en tant que» $z$. Inversement, tout acte de reconnaissance implique donc toujours une fêlure identitaire, une brisure que dans les mots de Bernhard Waldenfels on peut aussi qualifier de "différence récognitive", dans la mesure où celui ou celle qui se voit reconnu(e) n'est jamais identique avec celui ou celle en tant qu'il ou elle sera reconnu(e).

Pour illustrer cette donne, Thomas Bedorf fournit un exemple parlant: dans les préliminaires pour la conférence d'Annapolis sur le Proche Orient

23. Jessica Benjamin, Like Subjects, Love Objects : Essays on Recognition and Sexual Difference, New Haven, Yale University Press, 1998.

24. Charles Taylor 1994, « The politics of recognition », art. cit., p. 32.

25. Judith Butler, Undoing gender, New York, Routledge, 2004, p. 146. (Judith Butler, Défaire le genre, trad. Maxime Cervulle, Paris, Amsterdam, 2006, p. 171).

26. Ibid. 
en novembre 2007, le Premier ministre israélien Ehud Olmert avait déclaré que la reconnaissance d'Israël en tant qu'État juif constituerait le préalable non négociable pour la conférence ${ }^{27}$. Saeb Erekat, le négociateur en chef palestinien, répondit encore le jour même en affirmant que cette exigence était irrecevable, dans la mesure où "aucun État du monde ne relie son identité nationale à une identité religieuse ${ }^{28} »$. L'exemple illustre bien que ce qui est en jeu ici n'est pas l'existence factuelle d'un État nommé Israël - pas même les autorités palestiniennes ne le remettraient en question - mais bien un différend sur le sens et l'identité à lui attribuer. Précisément, le négociateur en chef Erekat n'affirme pas que l'Autorité palestinienne ne reconnaît pas Israël ; il affirme qu'elle ne reconnaît pas Israël en tant qu'État juif. Que la position palestinienne est loin de feindre une cécité pour l'état de fait, mais qu'elle conteste son interprétation, c'est ce que traduisent les mots d'Erekat: " aucun État du monde ne relie son identité nationale à une identité religieuse ». Il s'agit bien sûr ici avant tout d'un effet rhétorique qu'on ne saurait prendre trop à la lettre, car il suffit de penser à l'Arabie Saoudite, État religieux autoproclamé, qui refuse à tout juif de fouler son sol. Mais ici encore, l'interdiction porte sur l'institution d'une interprétation particulière : on pourrait imaginer le cas d'un touriste israélien ingénieur, socialiste, homosexuel et barbu auquel on interdirait l'entrée sur le sol saoudien. Or ce refus sera non pas motivé en vertu de son être homosexuel ou socialiste, mais uniquement en vertu de son être juif. Bref, un décalage existe au sein même de la reconnaissance qui se dédouble en plusieurs aspects.

\subsection{La reconnaissance en tant que mode de l'attention}

L'anglais nous permet de faire des distinctions dont le français ne dispose pas : il faudrait alors différencier entre acknowledgment et recognition, entre un terme bivalent et un terme trivalent. Si l'acknowledgment peut rester marginal, flou ou indéterminé (je remarque une présence, sans que je puisse déjà l'identifier ou sans que j'aie à l'identifier), la reconnaissance-recognition exige en revanche un acknowledgment préalable. Tandis que la recognition se laisse cerner plus facilement (elle est traduisible en une proposition du type $\mathrm{x}$ reconnaît $y$ en tant que $z$ ), le destin de l'acknowledgment demeure plus incertain. Car qu'est-ce à dire que $x$ reconnaît $y$, sans que cette reconnaissance implique une quelconque identification? Il semble qu'il faille débuter plus tôt encore, en postulant un état attentionel premier que l'on pourrait qualifier d'awareness, une attention pour ainsi dire à vide, une structure univalente donc du type $x$ est attentif.

27. Thomas Bedorf, Verkennende Anerkennung. Über Identität und Politik, Francfort, Suhrkamp, 2010, p. 118sq. A propos de la structure trivalente de la reconnaissance, cf. également Thomas Bedorf, « Le différend éthique », in : Alter. Revue de phénoménologie 13 (2005), p. 195-210.

28. Thomas Bedorf, Verkennende Anerkennung, op. cit., p. 119. 
Résumons les trois modes d'attention :

\begin{tabular}{|l|l|l|}
\hline Recognition & Structure trivalente & $x$ reconnaît $y$ en tant que $z$ \\
\hline Acknowledgment & Structure bivalente & $x$ remarque quelque chose $(y)$ \\
\hline Awareness & Structure univalente & $x$ est attentif \\
\hline
\end{tabular}

Il semble donc que la question de la reconnaissance, souvent débattue au niveau macroscopique, gagnerait à être réinsérée dans le contexte d'une micro-économie de l'attention sans laquelle tout acte de reconnaissance demeure abstrait et hors sujet. Pour le dire avec Waldenfels : " À l'origine, il n'y a pas quelqu'un (une personne, un sujet), à qui je dois quelque chose, mais quelqu'un devient pour moi quelqu'un à qui je dois quelque chose et à qui je prête ou je refuse quelque chose, notamment l'attention ${ }^{29}$. »

Parmi les théoriciens contemporains de la reconnaissance, certains ont tenté de situer les actes de reconnaissance dans leur enracinement somatique et social. Cette généalogie, entreprise notamment par Axel Honneth quand celui-ci tente de fonder sa théorie par des apports pédopsychiatriques, linguistiques et anthropologiques, reste toutefois précaire, aussi longtemps qu'elle n'aborde ces aspects que comme des étapes ancillaires sur le chemin de la négociation discursive. Comment donc concevoir que l'attention peut produire de la reconnaissance, sans que ce qui se voit ainsi reconnu ne se réduise à la confirmation d'une connaissance déjà constituée?

\section{L'inter-attention. Pour une éthique asymétrique}

Dans la Phénoménologie de la perception, Maurice Merleau-Ponty évoque la différence entre « une attention seconde » qui «se bornerait à rappeler un savoir déjà acquis » et une attention primaire dont l'objet ne saurait être anticipé $^{30}$. Cette distinction entre une attention première et une attention seconde, qui n'est pas sans résonner avec une autre distinction - celle entre parole parlante et parole parlée - installe d'emblée la problématique de l'attention sur un autre terrain que celui de la négociation des identités. Accorder la reconnaissance à quelqu'un ou encore la nier relèverait alors d'une attention secondaire, réflexive ou encore (pour parler avec Théodule Ribot) d'une attention "volontaire ${ }^{31}$ », tandis que l'attention primaire ne relève ni de notre initiative ni de notre vouloir. En reprenant les termes de Titchener, on peut dire que l'attention primaire « est une attention que nous sommes contraints d'accorder et que nous sommes incapables d'empêcher ${ }^{32}$ ». Le fait

29. Bernhard Waldenfels, Phänomenologie der Aufmerksamkeit, Francfort, Suhrkamp, 2004, p. 277. 30. Maurice Merleau-Ponty, Phénoménologie de la perception, Paris, Gallimard, 1945, p. 38. Sur l'enjeu de l'attention chez Merleau-Ponty, cf. Eran Dorfman, Réapprendre à voir le monde, Dordrecht, Springer, 2007, p. 57-63.

31. Théodule Ribot, Psychologie de l'attention [1889], Paris, L'Harmattan, 2007.

32. Edward Bradford Titchener, A Text-Book of Psychology, New York, Macmillan, 1910, p. 268. 
de détourner le regard ne fera que confirmer, après coup, qu'en premier lieu, je n'ai pu m'empêcher de regarder. Ma réponse, qu'elle soit affirmative ou négative, sera toujours seconde par rapport à ce qui aura suscité mon attention et jusque dans la dénégation, mon acte en certifiera l'efficace première. Dans ce cas, mon attention n'aura pas eu son origine en moi, mais résultera de mon être-interloqué par ce qui $\mathrm{m}^{\prime}$ aura surpris, étonné ou tout simplement intéressé. En s'appuyant sur Husserl, Bernhard Waldenfels évoque une «attention suscitée » par ce qui ne relève pas de mon ressort, une attention primaire qui précède une attention dirigée ou seconde. Une phénoménologie de l'attention qui prolongerait les riches analyses de Husserl semble donc apte à venir éclairer tout un pan des rapports intersubjectifs qui est comme effacé dans les théories de la reconnaissance.

Et pourtant, pour l'essentiel, ce travail reste à faire. Car bien qu'on trouve effectivement chez Husserl une réflexion féconde sur les multiples niveaux d'attention, notamment dans le volume XXXVIII de l'Husserliana ${ }^{33}$, sa phénoménologie de l'attention semble comme happée par le tourbillon de l'intentionnalité. Natalie Depraz a montré toute l'ambiguïté de Husserl à l'égard de la question de l'attention, décrite d'une part comme un projet à part entière qu'il s'agirait de mener à bien, et rabattue d'autre part à une simple modification de l'intentionnalité, elle-même soumise à une investigation eidétique ${ }^{34}$. Il $\mathrm{n}^{\prime} \mathrm{y}$ aurait dès lors d'attention que comme attention de quelque chose et d'attention de quelque chose qu'en tant qu'à travers les contingences de leur aspect, les choses permettent d'accéder à la chose en tant que chose, c'est-à-dire à leur essence ${ }^{35}$. Contrairement à des positions telles que celles de Ribot, Titchener ou Waldenfels, Husserl entend par «attention primaire» une attention dirigée vers la saisie de l'objet, tandis qu'une attention seconde est de l'ordre de l'apprésenté, de ce qui accompagne donc la visée comme pour ainsi dire " en écharpe ». L'ordre des priorités est donc clairement énoncé.

Or à faire remonter l'attentionnalité à l'intentionnalité et l'intentionnalité à l'eidétique, on risque de manquer que l'attention n'est pas faite uniquement de visées frontales, mais qu'elle procède elle-même par détours et que l'attention ne s'intensifie pas par la volonté ou l'effort, mais par une certaine disposition pour ce qui par définition ne se laisse pas anticiper.

33. Edmund Husserl, Wahrnehmung und Aufmerksamkeit. Texte aus dem Nachlass (1893-1912), éd. Thomas Vongehr et Regula Giuliani, Husserliana XXXVIII, Heidelberg, Springer, 2004 (fr. Phénoménologie de l'attention, introduction, traduction et notes par Natalie Depraz, Paris, Vrin, 2009).

34. Natalie Depraz, "Where is the phenomenology of attention that Husserl intended to perform? A transcendental pragmatic-oriented description of attention», Continental Philosophy Review. Special Issue on Attention 37 (A. Steinbock et N. Depraz eds.), 2004, p. 5-20. Cf. Natalie Depraz, "Attentionalité et intentionnalité. L'attention comme "modulation" », in : Husserl, sous la dir. de Jocelyn Benoist, Paris, Cerf, 2008, p. 223-248.

35. Natalie Depraz, «Where is the phenomenology of attention that Husserl intended to perform? », art. cit., p. 9sq. 


\section{L'attention}

\subsection{De l'attention comme relation à l'inanticipable (pour une éthique non objective)}

Plutôt que de venir vers les choses, l'attention les laisse advenir, mais ne les constitue pas. En empruntant les mots de Nietzsche, on peut rappeler que les idées viennent quand elles veulent, et non quand je le veux ${ }^{36}$. Ou pour prélever une formulation analogue chez William James, auteur qui marque un tournant important dans l'histoire de l'attention, retracée par Jonathan Crary: "Les choses viennent à nous d'après leur propre loi. L'attention ne crée aucune idée » (The things we attend to come to us by their own laws. Attention creates no idea ${ }^{37}$ ).

L'attention renvoie donc foncièrement à une extériorité inanticipable. Si elle implique que nous nous focalisions sur l'objet émergeant en nous tournant vers celui-ci, cette orientation est elle-même déjà seconde puisque suscitée par une stimulation qu'elle n'a pas elle-même produite. Levinas a montré comment, loin de se laisser subsumer sous l'intentionnalité, l'attentionalité dessaisissait plutôt celle-ci. L'attention, écrit-il dans Totalité et infini, c'est-à-dire ce qui semble correspondre à la souveraineté intérieure la plus complète, n'est jamais que réponse à un appel : «L'extériorité de son point de départ lui est essentielle, à elle, qui est la tension même du moi $^{38}$. » Ce que Levinas semble indiquer ici au détour d'une phrase, c'est rien de moins qu'une inversion des priorités husserliennes. Si on a pu tenter de lire dans l'intentionnalité une structure générale de tension qui excède de loin le contenu noématique et que l'on peut décliner comme motricité ou comme désir, Levinas fait signe ici vers une tensionnalité qui n'est plus le résultat d'un mouvement qui prend son origine dans le cogito, mais qui est déjà réponse. Loin d'être la modification de l'intention, c'est l'intention qui n'est qu'un mode de l'attention.

À cette attentionalité première, certains manuscrits husserliens semblent faire écho, quand l'intérêt se voit défini comme "direction vers quelque chose qui affecte ${ }^{39}$ » et dont l'affect précède dès lors l'acte d'orientation. L'attention est donc bien une tension active, mais pas encore «un agir proprement dit »: tandis que l'activité est dirigée vers un objet ou un but "déjà constitué ", l'attention relève d'une tension qui, comme le désir, se réfère à quelque chose qui sera inévitablement "manqué » car ayant

36. Friedrich Nietzsche, Jenseits von Gut und Böse I, § 17 (Sämtliche Werke, Munich-Berlin-New York 1999, KSA vol. 5, p. 31) «Ein Gedanke kommt, wenn "er" will, und nicht wenn "ich" will ».

37. William James, Principles of Psychology [1890], éd. Frederick Burckhardt, Harvard, Harvard University Press, 1981, chap. XI "Attention", p. 426. Cf. Jonathan Crary, Suspensions of perception. Attention, spectacle and modern culture, Cambridge, Mass., MIT Press, 2001.

38. Emmanuel Levinas, Totalité et Infini, La Haye, Nijhoff, 1961, p. 73.

39. Manuscrit datant de septembre 1931 environ. Edmund Husserl, Zur Phänomenologie der Intersubjektivität, Dritter Teil : 1929-1935, ed. Iso Kern, Hua XV, La Haye, Nijhoff, 1973, p. 329, appendice XIX (fr. Autour des "Méditations cartésiennes" (1929-1932): Sur l'intersubjectivité, trad. Natalie Depraz, Pol Vandevelde, Marc Richir, Grenoble, Jérôme Millon, 1998, p. 301). 
toujours « déjà disparu 40 ». En continuant ces réflexions, on pourrait donc avancer que phénoménologiquement, l'attention n'est nullement une contemplation détachée, elle est plutôt l'amorce d'une kinesthèse orientée qui se dirige vers ce qui la suscite originellement mais qui, comme stimulation originelle, ne se manifeste que dans l'après-coup de la réponse orientée. Il semble que la structure même de l'attentionnalité que de tels passages mettent au jour permette à Levinas de dépasser le cadre d'une intentionnalité dont l'ego aurait l'initiative en direction d'une intersubjectivité première. Cependant, l'intersubjectivité elle-même demeure chez Levinas fortement marquée par une scène dyadique, fût-elle asymétrique. «L'attention est attention à quelque chose » écrit-il ainsi " parce qu'elle est attention à quelqu'un ${ }^{41}$ ». Il y aurait non seulement à développer plus amplement la question de la place qu'accorde Levinas au Tiers, mais à s'interroger si la conception personnaliste du tiers ne n'empêche pas d'emblée qu'un événement, un objet ou une relation prenne la place $d u$ tiers.

\subsection{De l'attention comme mouvement en arc de cercle (pour une éthique non personnelle)}

Les recherches empiriques sur l'attention conjointe (joint attention) viennent ici confirmer que l'extériorité peut s'entendre autrement encore que comme l'extériorité de la face. Bien que nous subordonnions généralement la relation que nous entretenons avec les choses, les événements, et les détails $\mathrm{du}$ quotidien à la relation que nous entretenons avec autrui, celle-ci est pourtant souvent la base de celle-là. Dans la situation de l'attention conjointe dont Tran Duc Thao - le phénoménologue vietnamien injustement oublié disait avec une formule heureuse qu'elle est toujours «en arc de cercle ${ }^{42}$ », c'est l'unidirectionnalité du faisceau intentionnel qui se courbe et se plie pour former un espace attentionnel partagé. Loin de ne former donc qu'une étape dans un processus cognitif d'appropriation (de « remplissement » intentionnel), le phénomène de l'attention ouvre une scène intersubjective qui ne se ramène plus à l'économie de la propriété.

La situation de la joint attention ou attention conjointe est généralement inaugurée par un mouvement déictique ou d'indication (de deixis - deiknumi : montrer, indiquer, faire voir). Ainsi par exemple, c'est par un geste déictique que l'enfant attire l'attention de l'adulte et lui indique du doigt l'objet de son intérêt. Une tradition influente, allant de Wilhelm Wundt à Vygotsky, considère que ce geste déictique n'est que l'amorce d'un geste de préhension, l'enfant demandant à l'adulte de lui procurer ce qu'il ne peut

40. ibid.

41. ibid.

42. Tran Duc Thao, Recherches sur l'origine du langage et de la conscience, Paris, Editions Sociales, 1973, p. 26. 


\section{L'attention}

lui-même atteindre ${ }^{43}$. Les études de primatologie ont montré que l'on trouve également, parmi les grands singes, une grande variété de formes communicationnelles visant l'appropriation d'un objet (qu'il s'agisse d'une requête provenant du jeune chimpanzé ou au contraire de la guenon indiquant une source de nourriture à son petit, tout en prenant soin qu'il soit bien le seul à recevoir cette information ${ }^{44}$ ). Si l'existence de gestes strictement déictiques chez les grands singes - c'est-à-dire le fait de pointer sans communication vocale - fait débat parmi les primatologues ${ }^{45}$, ceux-ci $\mathrm{s}^{\prime}$ accordent toutefois sur le fait que tout geste n'aura toujours de valeur qu'appropriative, téléologique, bref: instrumentale. Pour le dire avec Tomasello : "Chimpanzees use and understand their gestures as one-way procedures for getting things done ${ }^{46}$.»

L'avancée des recherches en primatologie a permis de mieux dégager certaines perspectives développementales dans l'ontogenèse humaine. Contrairement à la conception qui faisait dériver l'indication d'un geste de préhension, le geste déictique que l'on observe tôt dans le développement des sujets humains renvoie souvent à une attention dont le partage même semble procurer à l'enfant un certain plaisir. Il faudrait dès lors distinguer entre deux types d'indications, une indication proto-impérative, consistant en une demande de l'objet qui peut être satisfaite dès lors que le sujet reçoit l'objet exigé, et une indication proto-démonstrative consistant à indiquer ce qui se tient dans un horizon commun, sans que cette démonstration ne donne lieu à une exigence d'appropriation ${ }^{47}$.

43. Wilhelm Wundt, Völkerpsychologie. Eine Untersuchung der Entwicklungsgesetze von Sprache, Mythus und Sitte, Leipzig, Engelmann, 1904, vol. 1 : Die Sprache, p. 136sq. Lev Vygotsky, Mind in Society: The development of higher psychological processes, Cambridge, MA : Harvard University Press, 1978, p. 56sqq.

44. Jane Goodall, The Chimpanzees of Gombe. Patterns of Behaviour, Cambridge, Belknap Press, 1986. David Leavens \& William Hopkins, "Intentional communication by chimpanzees. A crosssectional study of the use of referential gestures ", in : Developmental Psychology 34 (1998), p. 813822. Michael Tomasello \& Josep Call (ed.): The gestural communication of Apes and Monkeys, Mahwah, Erlbaum, 2007.

45. Les deux positions antinomiques dans ce débat étant respectivement celles de Michael Tomasello et de Frans De Waal (Frans De Waal, «Pointing primates: Sharing knowledge... without language ", in: Chronicle of Higher Education (janvier 2001), B7-B9. Michael Tomasello, «Why don't apes point? » In N. Enfield \& S. Levinson (eds.), Roots of Human Sociality, New York, Wenner-Grenn, 2006, p. 506-524).

46. Tomasello, «Why don't apes point? », art. cit., p. 515.

47. Luigia Camaioni, Virginia Volterra \& Elizabeth Bates, La comunicazione nel primo anno di vita, Torino, Bollati Boringhieri, 1976. Tomasello parle, lui, de « déclaratifs ». On peut se demander si les expressions « proto-démonstratif » et « déclaratif » ne concèdent pas trop déjà aux structures attributives du langage propositionnel, posées comme telos dans le développement de la communication intersubjective. On pourrait ainsi songer à les substituer, pour reprendre la suggestion de Natalie Depraz, par une « indication phatique » qui «fait voir » ce qui, en étant rendu visible, institue le lien de l'être-ensemble. 
Il semble bien que dans ce que Werner et Kaplan décrivaient comme la "situation primordiale de partage " (primordial sharing situation) ${ }^{48}$, l'enfant tire du simple fait d'attirer l'attention de l'adulte sur un objet ou un événement une jouissance qui n'implique aucune possession et aucune propriété, un être-adonné ou encore une présence-à, exempts de toute prise. Tout semble indiquer que les grands singes sont capables de deux attitudes distinctes: a) du geste proto-impératif de monstration, demandant à un autre sujet (animal ou humain) d'obtenir un objet pour eux et b) de dépenser du temps dans une attitude d'attention réciproque (dans l'acte d'épouillement par exemple), mais les deux attitudes ne convergent pas dans une situation d'attention conjointe, incapacité dont font preuve également les enfants autistes ${ }^{49}$.

Investir philosophiquement les recherches sur la primatologie et l'hominisation ne revient pas nécessairement à postuler une nouvelle différence anthropologique, où l'essence humaine serait définie non plus par son être doué de langage ou de la faculté symbolisation, mais par la faculté à partager un espace attentionnel. Loin de ne former donc qu'une étape dans un processus cognitif d'appropriation (de « remplissement » intentionnel), le phénomène de l'attention engage un être-ensemble qui ne produit que du commun, mais rien de propre. La différence des résultats sur les singes en nature et en captivité montre ici l'importance du milieu. Contre l'ontologisation de Tomasello, David Leavens et d'autres ont insisté sur la spécificité du milieu humain. C'est alors non plus en vertu d'une essence anthropologique, mais bien plutôt à cause d'un environnement différent qu'émerge le phénomène de l'indication. Les études sur l'«espace référentiel » prouvent que si les singes en captivité font preuve de gestes d'indication, c'est non pas tant en vertu de leur prétendu «hominisation » au contact avec des sujets humains, mais parce qu'ils partagent avec un nouveau-né la même situation de limitation: comme le nourrisson dans la crèche, le singe derrière les barreaux n'a guère d'accès direct aux choses et développe donc avec celles-ci un rapport indirect qui passera par le geste d'indication ${ }^{50}$.

Quoi qu'il en soit, repartir de la scène de l'attention conjointe pour concevoir les enjeux de l'intersubjectivité - partir donc de ce que Merleau-Ponty nomme la «co-perception ${ }^{51}$ » - c'est ne pas faire débuter le questionnement éthique au niveau des exigences discursives et des

48. Heinz Werner \& Bernard Kaplan, Symbol Formation. An Organismic-Developmental Approach to Language and the Expression of Thought, New York, Wiley, 1963.

49. Juan Carlos Gomez, "Joint Attention and the Notion of Subject: Insights from Apes, Normal Children, and Children with Autism » in : Joint attention. Communication and other minds, sous la dir. de Naomi Eilan, Christopher Hoerl, Teresa McCormack \& Johannes Roessler, Oxford, Oxford University Press, 2005, p. 65-84.

50. David Leavens, William Hopkins \& Kim Bard, «The heterochronic origins of explicit reference", in: The shared mind. Perspectives on intersubjectivity, ed. Jordan Zlatev et al., Amsterdam, John Benjamins, 2008, p. 187-214.

51. Maurice Merleau-Ponty, Signes, Paris, 1960, p. 215. 


\section{L'attention}

demandes explicites, mais au niveau de l'enracinement somatique et sensible de l'être-ensemble, bref : dans l'intercorporéité. Contrairement à la scène hégélienne de la reconnaissance réciproque, le lien commun ne se fait pas sur le mode d'un être-l'un-par-l'autre, mais par l'être adonné à ce qui ne relève ni de l'autre ni du Moi.

\subsection{Donner le temps, donner ce que l'on n'a pas (pour une éthique de l'impropre)}

Dans sa théorie de la reconnaissance, Axel Honneth précise que la reconnaissance ne saurait se réduire à une connaissance. Néanmoins, l'acte de reconnaissance reste toujours d'une part un acte et de l'autre un acte rétributif: en reconnaissant de la valeur à autrui, je procède à une «perception évaluative ${ }^{52}$ », c'est-à-dire je lui accorde une valeur spécifique, comparable à la valeur que je m'accorde moi-même. Cette comparaison produit à son tour un tertium comparationis, constitué à partir de ce qui est propre à nos deux identités. Les éthiques de la reconnaissance et l'éthique de l'attention se distinguent notamment en ceci que cette dernière part d'un commun qu'aucun ne possède et qui est pourtant partagé, elle ne vise pas la reconnaissance d'une propriété quelconque mais se tient dans la stricte limite de la dépense. La dépense de quelque chose que le sujet ne peut prêter que parce que lui-même ne fait que l'emprunter : l'attention.

Nous sommes certes ici aux antipodes de ce que la tradition philosophique projetait dans l'attention, c'est-à-dire une technique de focalisation, de recentrement, de concentration. Être attentif, en ce sens, «c'est être centré sur soi et se focaliser sur l'objet pour en identifier les propriétés ${ }^{53}$ ». Quel que soit l'angle de vue : c'est toujours une logique du propre qui est implicitement appelée, par opposition à une attitude de dispersion, de distraction et de «manque d'attention». Indépendamment du fait que cette opposition rate complètement la dimension modulatrice de l'attention - dans certaines situations comme dans la cure analytique par exemple, l'attention flottante pourra être plus à même de «saisir » ce dont il en va -, elle ne confirme qu'à rebours le préjugé de la réflexivité qui conditionne depuis toujours le rôle de l'attention en philosophie. Dans cette perspective, l'attention n'aura droit de cité qu'à condition d'amener à une plus haute conscience des propriétés, que ce soit sur le mode d'un retour sur soi ou d'une saisie des objets ${ }^{54}$.

À la différence du paradigme de la reconnaissance, qui permet que des identités se constituent en parties, mais aussi à la différence d'une tradition intellectualiste de l'attention en philosophie, une éthique de l'inter-attention

52. Axel Honneth, «Invisibilité : sur l'épistémologie de la reconnaissance », trad. F. Gollain et C. Lazzeri, in : Revue du MAUSS, «De la reconnaissance », n²3, 2004, p. 137-151, ici p. 149.

53. Natalie Depraz, «Introduction » à Husserl, in : Phénoménologie de l'attention, op. cit., p. 58.

54. Michael Hagner a retracé certains liens entre l'émergence de la question de l'attention et la pédagogie bourgeoise libérale du XIXe siècle («Toward a history of attention in culture and science », MLN 118 (2003), p. 670-687). 
se démarque d'une logique constitutive et concentrante pour aller en direction d'une pensée de la déprise. Le bref échange verbal lors d'un voyage en train décrit par Charles Taylor ${ }^{55}$ n'a rien d'une logique de la reconnaissance : si un des deux voyageurs du compartiment constate que la chaleur est étouffante aujourd'hui, il ne fait que verbaliser ce que l'autre sait déjà pertinemment. Ses mots n'ont pas pour but de transmettre une information ni même une requête de reconnaissance personnelle, ils attirent l'attention sur ce qui relie les deux voyageurs, sur cet «entre nous ». La situation conjointe repose sur le partage d'un milieu commun.

Accorder son attention n'est donc pas seulement le fruit d'une évaluation ou d'un choix; il est tout autant le résultat d'une mise en rapport précédant toute initiative de ma part. Je peux choisir de $\mathrm{m}^{\prime}$ ' accorder » à l'autre ou de me désaccorder, mais même en feignant de pas l'entendre - et l'autre peut être ici personne, animal, objet, événement -, mon silence délibéré ne fait que confirmer, a contrario, l'attention qu'il a suscité en moi. En ce sens, accorder son attention consiste bien à donner ce que nous n'avions jamais en propre. "To pay attention»- l'expression dit bien que toute «conversion attentionnelle » (Husserl) constitue toujours un mouvement de retour ou de restitution, tentative imparfaite pour combler une dette dont on ne peut pourtant, en toute rigueur, espérer un jour s'acquitter. Accorder son attention, $\mathrm{c}^{\prime}$ est donc bien : donner le temps ${ }^{56}$. Donner, dès lors, ce qui résiste à son économisation, à sa conversion, à sa rétribution. Donner, au fond, ce qui ne quittera jamais nos mains pour la simple raison qu'il ne saurait jamais y être, entre nos mains.

55. Charles Taylor, Human agency and language, Cambridge, Cambridge University Press, 1985, p. 264sq.

56. Jacques Derrida, Donner le temps 1. La fausse monnaie, Paris, Galilée, 1991. 